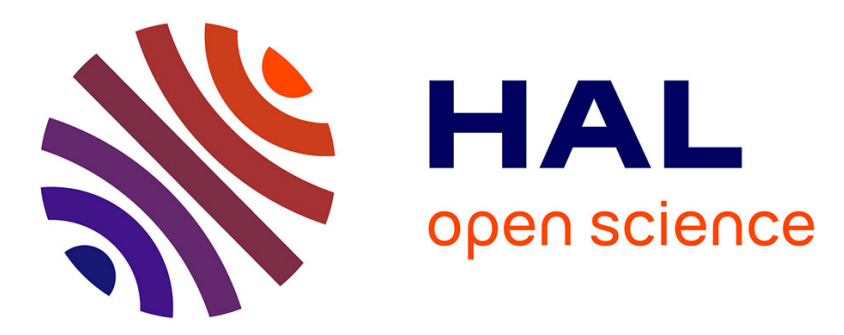

\title{
Production rapide d'un vide assez avancé à l'aide de la chaux. Quelques effets - De la décharge électrique dans les tubes a vapeur d'eau
}

\author{
A. Henry
}

\section{- To cite this version:}

A. Henry. Production rapide d'un vide assez avancé à l'aide de la chaux. Quelques effets - De la décharge électrique dans les tubes a vapeur d'eau. J. Phys. Theor. Appl., 1909, 8 (1), pp.33-38. 10.1051/jphystap:01909008003301 . jpa-00241463

HAL Id: jpa-00241463

https://hal.science/jpa-00241463

Submitted on 1 Jan 1909

HAL is a multi-disciplinary open access archive for the deposit and dissemination of scientific research documents, whether they are published or not. The documents may come from teaching and research institutions in France or abroad, or from public or private research centers.
L'archive ouverte pluridisciplinaire HAL, est destinée au dépôt et à la diffusion de documents scientifiques de niveau recherche, publiés ou non, émanant des établissements d'enseignement et de recherche français ou étrangers, des laboratoires publics ou privés. 


\section{PRODUCTION RAPIDE D'UN VIDE ASSEZ AVANCÉ A L'AIDE DE LA GHAUX. QUELQUES EFFETS}

DE LA DÉCHARGE ÉLEGTRIQUE DANS LES TUBES A VAPEUR D'EAU;

Par M. A. HENRY.

I. Il est possible, sans trompe à mercure, d'atteindre rapidement un vide assez avancé; il suffil de posséder une machine pneumatique ordinaire ou une trompe à eau, à la condition de recourir, comme auxiliaire, à la vapeur d'eau.

En principe, on remplit de vapeur d'eau pure le récipient où l'on veut faire le vide; puis on absorbe celle-ci par un corps bien choisi.

Parmi les nombreux procédés possibles, l'un des plus commodes consiste à prendre la chaux éteinte comme générateur de vapeur d'eau, et son résidu, la chaux vive, comme absorbant.

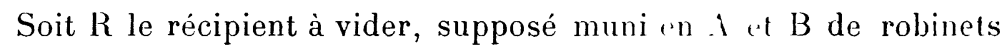
bien travaillés. Il est mis en communication du còti $\Lambda$, fig. 1) avec un tube de verre mince dont le diamètre est de 3 centimetres environ, contenant à peu près 20 grammes de chaux éteinte et du côté $B$ arec un autre tube de même diamètre, rempli de potass' caustique sur une longueur de 10 centimètres. Ce tube à putasse est relié al la machine pneumatique ou à la trompe à eau.

J. de Phys., 4' série. t. VIII. (Janvir. 1909.) 
Tandis que fonctionne la machine pneumatique ou la trompe à eau, on élève la température du tube à chaux avec une lampe à alcool. La vapeur d'eau se produit en abondance et balaye le récipient $\mathrm{R}$; afin de l'empêcher de transporter de la chaux, il est bon de placer avant l'extrémité $A$ un tampon d'amiante.

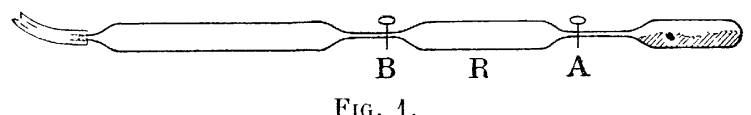

Après quelques minutes de chauffe, on ferme le robinet $B$ et on laisse l'appareil se refroidir. La chaux réabsorbe la vapeur d'eau en un quart d'heure environ. Il suffit de fermer le robinet $\Lambda$ et de détacher le tube à chaux; on a ainsi un vide assez avancé. A défaut de robinets, les extrémités A et B seront étirées au préalable, afin que . l'on puisse les fermer à la lampe dans l'ordre indiqué.

Dans l'un des essais, $R$ avait la forme d'un long tube barométrique ayant $2^{\mathrm{rm}}, \check{\jmath}$ de diamètre, il fut fermé à la lampe, puis ouvert sur une cuve à mercure bien pur. En comparant la hauteur mercurielle ̀̀ celle d'un bon baromètre Tonnelot, on a évalué la pression dans la chambre barométrique. En ramenant la pression à ce qu'elle était, lorsque la vapeur était répandue dans tout le volume, on a trouvé $2 / 10$ de millimètre. Le résultat donné par comparaison avec le baromètre Tonnelot a été vérifié en réduisant de moitié le volume de la chambre barométrique du tube à vapeur d'eau. D'ailleurs cette vapeur pendant ces expériences était loin d'être saturante.

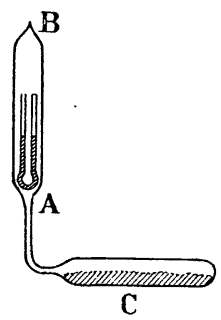

Fig. 2.

Cette pression peut différer suivant la quantité d'eau perdue par la chaux; l'aspect de la décharge électrique dans des tubes ainsi vidés le montre nettement. 
On peut le mettre en évidence aussi en prenant le tube représenté par la fig. 2. C renferme la chaux et $\mathrm{AB}$ contient un manomètre à mercure. Le vide y est réalisé comme plus haut. Mais si la fermeture en $\mathrm{B}$ a été faite après un léger chauffage de $\mathrm{C}$, il suffit de réchauffer le tube à chaux même légèrement pour voir reparaître une pression; deux tubes préparés à peu près dans les mêmes conditions n'ont pas donné à $100^{\circ}$ la mème pression. Si la fermeture suit un chauffage plus prolongé, l'absorption est plus rapide; puis le tube porté à $250^{\circ}$ ne donne pas d'accroissement de pression. En le soumettant de nouveau à la flamme de la lampe à alcool, une pression reparaît. De tels tubes permettront de voir si la chaux éteinte possède une tension de dissociation. Quoi qu'il en soit, pour obtenir le vide le plus avancé, on se placera dans le deuxième cas.

Dans ces opérations le verre n'est pas attaqué par la chaux. Cette dernière peut se régénérer en l'abandonnant à l'air humide. Ces expériences ont été faites avec la chaux du marbre. La baryte hydratée conduit à des résultats analogues; mais elle émet des gaz dans le vide, qui font perdre au tube à vapeur d'eau ses propriétés ; la chaux est donc préférable sous ce rapport.

Le vide atteint correspondrait par litre à une masse de vapeur d'eau inférieure à $1 / 3$ de milligramme.

Les balances sensibles ordinaires présentent cette limite de sensibilité ; par suite, dans bien des expériences, on pourrait considérer le récipient comme vidé parfaitement.

11. Ces tubes à vapeur d'eau, que l'on peut préparer en quelques minutes, conviennent bien pour l'enseignement, quand il s'agit de montrer certains effets de la décharge électrique.

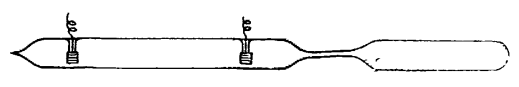

FIG. 3.

Soit un tube à deux électrodes d'aluminium encore solidaire du réservoir à chaux où le vide est réalisé ( $f g .3)$.

La décharge d'une bobine de Ruhmkorff provoque à l'opposé de la cathode une belle fluorescence jaune verdàtre, la tache se déplace à l'approche d'un aimant. Ces tubes donneraient donc des rayons cathodiques comme lampoule de Crookes. Ils permettent de réaliser 
l'expérience de la croix portant ombre sur le verre et d'autres se rapportant aux rayons cathodiques. Il suffit pour cela d'une petite bobine de Ruhmkorff donnant environ 1 centimètre d'étincelle.

Veut-on manifester les différents aspects de la décharge dans le mème tube, on élève progressivement la température de la chaux; la fluorescence diminue peu à peu, puis des strates envahissent le tube, qui présente ensuite l'aspect des tubes de Geissler.

En abandonnant l'appareil à lui-mème, en moins de cinq minutes, les mèmes apparences se succèdent en sens inverse.

Un long tube sans électrode, vidé de même, s'illumine dans toute sa longueur quand on le présente à l'un des pôles d'un petit transformateur de Tesla actionné par la bobine précédente. Chose curieuse : la partie du verre opposée à l'extrémité du fil du secondaire prend une belle fluorescence jaune verdâtre; cette tache est déplacée par l'aimant; même résultat avec l'ampoule de Crookes; mais aucune fluorescence jaune avec les lampes à incandescence. il faudrait donc admettre que le vide dans le tube à vapeur d'eau dépasse celui des lampes à incandescence et qu'il atteint le 1/1000 de millimètre environ. Cette conclusion est en contradiction avec la mesure de pression faite précédemment. Dès lors se présente l'hypothèse que les rayons cathodiques offrent dans la vapeur d'eau les mêmes aspects que dans l'ampoule de Crookes à des pressions bien supérieures à celles que l'on a indiquées jusqu'jci pour d'autres gaz. Dans le but de le vérifier, le tube de la fig. 2 est chaulfé jusquà ce que la pression dépasse 1 millimètre de mercure, puis il est présenté au pôle du transformateur : la tache jaune verdâtre apparaît encore; la pression est accrue peu à peu: la tache va en s'affaiblissant; mais elle ne disparaît définitivement que vers ă millimètres de mercure.

Dans ces tubes naissent bien des rayons cathodiques, la vérification en est faite à l'aide du tube focus (fig. 4) préparé de la même manière.

Le verre acquiert une fluorescence jaune verdàtre; les rayons sont déviés par l'aimant; ils donnent au carbonate de calcium placé dans l'ampoule une belle fluorescence rose. Leur choc contre une lame de platine provoque la formation de rayons $\mathrm{X}$. Le tube précédent, aminci en face de la lame de platine, a permis de décharger rapidement un électroscope; il donne une belle fluorescence au platino-cyanure de baryum, enfin il impressionne une plaque photographique enveloppée de papier noir. 
Dans ce tube on peut augmenter la pression de la vapeur d'eau en élevant la température de la chaux: la fluorescence du verre devient moins vive, les rayons $\mathrm{X}$ sont affaiblis; mais l'aspect général de la décharge reste le même. La fluorescence persiste encore au-dessus de 2 millimètres.

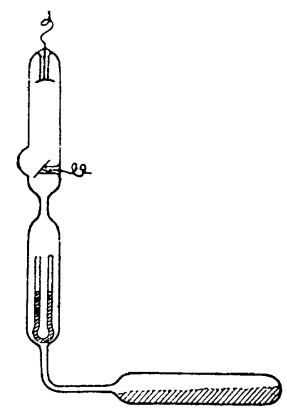

Firi. '.

Il est donc démontré que, dans la vapeur d'eau, à une pression d" l'ordre du millimèlre de mercure, on peut obtenir des phénsmines analogues à ceux que présente l'ampoule de Crookes.

Ces tubes offrent au passage de la décharge une faible résistance comparativement à celle des ampoules de Crookes. Pour la même distance d'électrodes, la décharge ne traversait le tube de Crookes que sous une différence de potentiel correspondant à $4^{\mathrm{cm}}, \hat{\partial}$ d’ètincelle, tandis qu'elle passait dans le tube à vapeur d'eau dès 2 millimètres d'étincelle.

Cette faible résistance est encore mise en évidence par un tube sans électrode d'une longueur de $\mathrm{I}^{\mathrm{m}}, \breve{30} 0$; tenu par un bout et présenté par l'autre extrémité à un pôle du transformateur, donnant 4 centimètres d'étincelle, il s'illumine dans toute sa longueur. Comme on n'utilise ainsi que la moitié de la différence de potentiel aux pôles du transformateur, on peut prévoir qu'un tube en forme d'arc placé entre les pôles s'illuminerait sur une longueur d'au moins 3 mètres.

Dès les premiers instants le tube ne s’illumine pas; il est nécessaire de produire une ionisation à l'aide d'un champ puissant, par exemple celui du transformateur de Tesla.

Les propriétés des tubes à vapeur d'eau sont assez surprenantes. Sont-elles dues à l'existence d'oxygène, d'ozone et d'hydrogène consécutifs de la décharge? 
Le rayon parti de la cathode serait-il entretenu dans sa marche par une espèce d'énergie chimique?

L'ozone se forme bien pendant la décharge; car le mercure du manomètre se recouvre d'une couche noire d'oxyde.

On constate que l'aluminium se couvre à la longue d'un enduit blanc très peu conducteur, qui parait être de l'alumine. Ces derniers effets sont surtout très nets, quand la décharge se produit sous une pression voisine du millimètre.

D'autres recherches seront poursuivies sur ces tubes et sur d'autres remplis de fluides différents. 